\title{
Transient Growth Factor Delivery Sustains Regenerated Axons after Spinal Cord Injury
}

\author{
Armin Blesch ${ }^{1}$ and Mark H. Tuszynski ${ }^{1,2}$ \\ ${ }^{1}$ Department of Neurosciences, University of California, San Diego, La Jolla, California 92093, and ${ }^{2}$ Veterans Administration Medical Center, San Diego, \\ California 92165
}

Growth factors influence the topography of axonal projections during nervous system development and facilitate axonal sprouting and regeneration after injury in the adult. However, in the absence of target reinnervation and reestablishment of synaptic activity, we hypothesized that continuing delivery of neurotrophins would be required to sustain regenerating axons for prolonged times points after neurotrophin-induced axon growth after spinal cord injury (SCI) in the adult. Using tetracycline-inducible expression of brain-derived neurotrophic factor by genetically modified fibroblasts, we were able to extensively and significantly turn growth factor expression "on" or "off" in vitro and in vivo within sites of SCI. Notably, we find that transient growth factor delivery is sufficient to sustain regenerated axons for prolonged time periods within spinal cord lesion sites. Immunohistochemical analysis demonstrated an absence of neuronal targets or synapses within transient growth factor expressing grafts but the persistent presence of Schwann cells. Thus, the adult CNS appears capable of sustaining axons that have extended after transient growth factor delivery, an effect potentially attributable to the persistence of Schwann cells in lesion/graft sites.

Key words: gene therapy; neurotrophin; spinal cord injury; tetracycline; regulated gene expression; regeneration; Schwann cells; BDNF

\section{Introduction}

Investigations from a number of laboratories have demonstrated that the delivery of neurotrophic factors to the injured spinal cord can induce extensive axonal growth from ascending and descending axonal populations (Xu et al., 1995; Tuszynski et al., 1996, 1999; Grill et al., 1997; Kobayashi et al., 1997; Ye and Houle, 1997; Bradbury et al., 1998, 1999; Jakeman et al., 1998; Menei et al., 1998; Blesch et al., 1999; Bunge and Kleitman, 1999; Houle and Ye, 1999; Liu et al., 1999; Jin et al., 2000; Ramer et al., 2000; Bamber et al., 2001; Coumans et al., 2001). Cells producing nerve growth factor (NGF), brain-derived neurotrophic factor (BDNF), neurotrophin-3 (NT-3), NT-4/5, leukemia inhibitory factor, and glial cell line-derived neurotrophic factor promote specific regenerative responses after grafting to rats with spinal cord injury (SCI) (Tuszynski et al., 1994; Grill et al., 1997; Blesch et al., 1999, 2004; Liu et al., 1999; Himes et al., 2001; Lu et al., 2001, 2005; Jin et al., 2002; Blesch and Tuszynski, 2003; Tobias et al., 2003).

Previous studies investigating axonal growth responses to cellular neurotrophin delivery in the injured spinal cord used constitutively active promoters to express the transgene. Although there is a slight decline in gene expression over time, neurotrophic factor expression continues for several months to years (Tuszynski et al., 1996; Blesch and Tuszynski, 2003; Blesch et al.,

Received April 26, 2007; revised Aug. 9, 2007; accepted Aug. 16, 2007.

This work was supported by grants from the International Spinal Research Trust, California Roman Reed Spinal Cord Injury Fund, Wings for Life, Veterans Administration, Adelson Medical Research Foundation, and National Institutes of Health Grants NS46466, NS54833, NS047101, and NS09881.

Correspondence should be addressed to Dr. Armin Blesch, Department of Neurosciences-0626, University of California, San Diego, 9500 Gilman Drive, La Jolla, CA 92093-0626. E-mail: ablesch@ucsd.edu.

DOI:10.1523/JNEUROSCI.1903-07.2007

Copyright $\odot 2007$ Society for Neuroscience 0270-6474/07/2710535-11\$15.00/0
2004), leading to extensive axonal growth into, but not beyond, the lesion and graft site. As mentioned above, responses of adult axons to increases in neurotrophin concentration in the spinal cord have been investigated in detail, but axonal responses to subsequent reductions in growth factor support have not been characterized to date. Declining trophic factor concentrations at the lesion site could result in retraction of axons that have extended into a cellular neurotrophic factor producing graft. Such a response would indicate that axonal growth into a spinal cord lesion and retention of axons in the graft are equally dependent on high neurotrophic factor levels. Alternatively, if axonal growth is dependent on high neurotrophin levels but retention of axons is independent of high trophic factor levels, transient neurotrophic factor delivery would be sufficient to induce axonal growth and to retain axons for an extended time period in a lesion site.

During nervous system development, transient growth factor gradients help to establish the topography of target innervation (Tessier-Lavigne and Goodman, 1996; Tucker et al., 2001; Ma et al., 2002; Markus et al., 2002; Marotte et al., 2004). Once axons reach appropriate target regions, synapses form and newly developing circuits establish neural activity. Active projections tend to be sustained, whereas inactive projections are pruned, potentially by a "Hebbian mechanism" that contributes to patterning of the nervous system (Hebb, 1949). By extension to the injured adult CNS, one would hypothesize that postlesion sprouting or regeneration of axons, whether natural or experimentally induced, would likely be sustained only when functional projections are established or if a continuous stimulus for growth is present (such as a growth factor). To address this hypothesis, we used tetracycline-regulatable systems (Gossen and Bujard, 1992; Gossen et al., 1993, 1995) to control the duration of BDNF gene 
delivery to sites of midcervical spinal cord injury. The regulated expression of growth factors is both essential to testing the hypothesis of this experiment and to optimize the safety of gene delivery in a variety of models of CNS disease (Zoltick and Wilson, 2001; Maguire-Zeiss and Federoff, 2004). Using an improved tetracycline-inducible system in a single retroviral vector, we now report the ability to "tightly" control BDNF protein levels in the injured adult CNS using an orally delivered regulator. Furthermore, we find that transient growth factor delivery to a site of CNS injury is sufficient to sustain long-term axonal projections.

\section{Materials and Methods \\ Vector construction and retroviral vector production}

The basis for the retroviral vector construction was the previously described plasmid pLN-tetoff (Blesch et al., 2000, 2001). First, we removed the tetracycline transactivator ( $\mathrm{tTA}$ ) coding sequence and the internal simian virus 40 polyadenylation site (SV40 polyA) from pLN-tet-off by $E c o$ RI/BglII digestion and replaced it with the bovine growth hormone polyadenylation signal (BGH polyA). Unlike the SV40 polyA signal, the BGH polyA signal is only fully functional in one direction and was cloned in antisense direction into the vector behind the tetracycline-regulated cytomegalovirus (CMV) promoter. The resulting plasmid was digested with $E c o$ RI and an EcoRI fragment containing the coding sequence for the reversed tetracycline transactivator rtTA2s-M2 (Urlinger et al., 2000) was inserted resulting in the new vector pLN-teton. A NotI/BglII fragment containing the coding sequence for enhanced green fluorescent protein (GFP) was cloned in antisense direction between the regulatable CMV promoter and the BGH polyA site into pLN-tet-on. For the regulatable BDNF vector, a NotI/Bam HI fragment containing the human BDNF cDNA (gift from L. Reichardt, University of California, San Francisco, CA) was cloned into the NotI/BglII digested pLN-tet-on (Fig. $1 \mathrm{~A}$ ).

Retroviral vectors were produced by transient transfection of pLNtet-on-BDNF and pLN-tet-on-GFP into Phoenix ampho retrovirus producer cells using $\mathrm{CaPO}_{4}$ transfection (Pear et al., 1997). Supernatants were harvested after $48 \mathrm{~h}$ and stored at $-80^{\circ} \mathrm{C}$. Cultures of primary rat fibroblasts (see below) were infected with supernatants from the Phoenix producer cells. Transfected cells were selected by adding G418 (400 $\mu \mathrm{g} /$ $\mathrm{ml})$ to the cell culture medium.

\section{Cell culture}

Cultures of primary fibroblasts were obtained from skin biopsies and cultivated under standard cell culture conditions in DMEM/10\% FBS as described previously (Blesch et al., 1999). Briefly, $2 \times 2 \mathrm{~cm}$ skin biopsies obtained from the abdominal region of adult Fischer 344 rats were cut into small pieces $(1 \times 1 \mathrm{~mm})$ and were placed under a glass coverslip attached to a cell culture Petri dish with silicone grease. Biopsies were incubated in cell culture medium for 2-4 weeks until fibroblasts migrated from the skin biopsies onto the surrounding cell culture Petri dish. Cells were split 1:3 shortly before reaching confluency. Cells from low passage numbers (less than four) were used for retroviral infection and in vitro characterization and in vivo grafting.

In vitro characterization of transduced fibroblasts

Dose-response curves. tet-on-BDNF transduced fibroblasts were seeded into 12 -well plates $\left(5 \times 10^{4}\right.$ cells per well in $1 \mathrm{ml}$ of cell culture medium and pretreated for $48 \mathrm{~h}$ with doxycycline (Dox) with different concentrations $(0,0.01,0.1,1,10,100$, and $1000 \mathrm{ng} / \mathrm{ml}$; three wells for each concentration). The cell culture medium was replaced with fresh medium containing the same concentration of Dox as in the preincubation, and supernatants were collected $24 \mathrm{~h}$ later for analysis of BDNF levels by ELISA.

Time course. tet-on-BDNF transduced fibroblasts were seeded into 12 -well plates $\left(5 \times 10^{4}\right.$ cells per well) and incubated for $48 \mathrm{~h}$ when they reached confluency. The medium was replaced with fresh medium, six wells received no doxycycline, and six wells were treated with $1 \mu \mathrm{g} / \mathrm{ml}$ doxycycline. Twenty-four hours later, medium was replaced $12 \mathrm{~h}$ before the start of the assay with $1 \mathrm{ml}$ of cell culture medium with or without doxycycline as in the previous incubation. At time 0 , the first $12 \mathrm{~h}$ supernatant was collected, cells were washed three times with $4 \mathrm{ml}$ of cell culture medium, and cells received new medium. One group continued to receive no doxycycline, one group continued to be cultivated with doxycycline $(1 \mu \mathrm{g} / \mathrm{ml})$, one group was changed from medium without doxycycline to medium with doxycycline, and one group was changed from medium with doxycycline to medium without doxycycline. At time points of $12,24,36,48,60$, and $72 \mathrm{~h}$, medium was changed and collected for ELISA analysis of BDNF levels.

\section{BDNF ELISA}

Ninety-six-well plates were coated with a rabbit anti-BDNF antibody (gift from Amgen, Thousand Oaks, CA) in carbonate buffer using normal rabbit IgG as control. After several washes, plates were blocked with $3 \%$ bovine serum albumin (BSA), and serial dilutions of cell culture supernatants or tissue lysates from animals grafted with tet-on-BDNF 

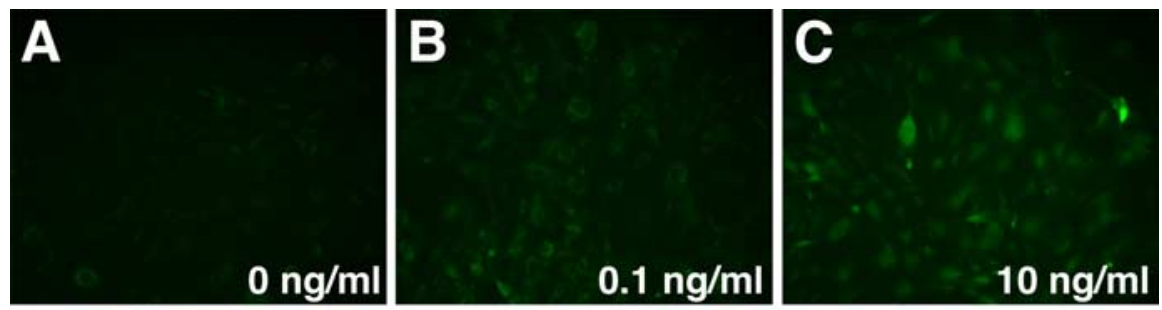

Health and Society for Neuroscience guidelines for animal care were strictly followed.

\section{Lesion surgery}

Rats were deeply anesthetized with a mixture (3 $\mathrm{ml} / \mathrm{kg})$ of ketamine $(25 \mathrm{mg} / \mathrm{ml})$, rompun $(1.3$ $\mathrm{mg} / \mathrm{ml})$, and acepromazine $(0.25 \mathrm{mg} / \mathrm{ml})$. T7 dorsal laminectomies were performed, and subjects received T7 spinal cord dorsal hemisection lesions $(n=120)$.

To create dorsal hemisection lesions, the dorsal half of the spinal cord was aspirated using a fine-tipped glass-pulled device, as described previously (Grill et al., 1997; Blesch et al., 1999). Immediately after the lesion, animals received implants that filled the lesion cavity of syngenic fibroblasts expressing BDNF or GFP as control under the control of the tetracyclineinducible promoter. Grafted fibroblasts were embedded in a matrix of liquid collagen (Sigma, St. Louis, MO) as described previously (Blesch et al., 1999) to prevent their leakage away from the lesion site. Muscle layers were sutured, and the skin was closed with staples. Four animals

Figure 2. In vitro characterization of tet-on-GFP transduced fibroblasts. Fluorescence of fibroblasts transduced with retroviral vectors for the tetracycline-inducible expression of green fluorescent protein $36 \mathrm{~h}$ after addition of Dox in vitro. Intensity of GFP fluorescence increases with increasing concentrations of Dox, reaching a maximum at $100 \mathrm{ng} / \mathrm{ml}$ Dox. Concentrations of Dox are indicated in each panel. Scale bar, $134 \mu \mathrm{m}$.
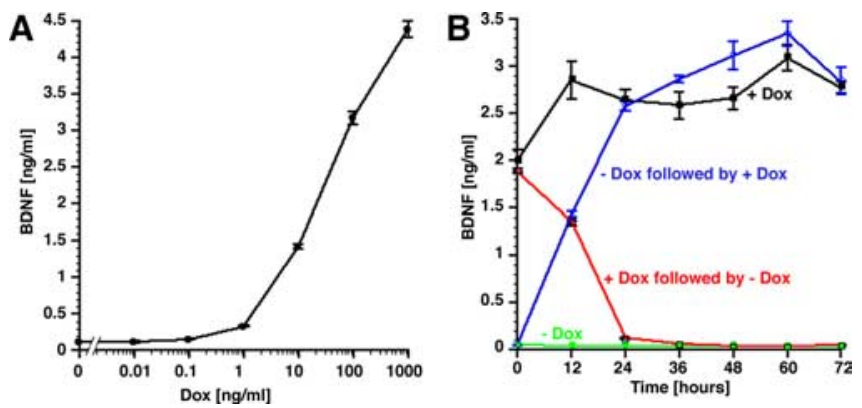

Figure 3. Dose dependence and kinetics of BDNF production in primary Fischer 344 rat fibroblasts transfected with tet-on-BDNF. $A$, With increasing concentrations of doxycycline in the cell culture medium, BDNF expression is increased. $\boldsymbol{B}$, Kinetics of BDNF expression. tet-onBDNF transfected cells were cultivated for $3 \mathrm{~d}$ with doxycycline (red and black lines) or without doxycycline (blue and green circles). Supernatants were collected beginning at time 0 . One group (red line) was changed from doxycycline-containing to doxycycline-free medium, and one group (blue line) was changed from doxycycline-free to doxycycline-containing medium. BDNF levels were measured by ELISA. Cells that were constantly cultivated in doxycycline-free medium (green line) showed very little BDNF expression. Cells that were constantly treated with $1 \mu \mathrm{g} / \mathrm{ml}$ doxycycline (black line) showed continued BDNF expression. Cells that were first in doxycycline-free medium and were then changed to doxycycline-containing culture medium at time 0 (blue line) turned on gene expression within $12 \mathrm{~h}$. This was evidenced by the $24 \mathrm{~h}$ supernatant (collected between the 12 and $24 \mathrm{~h}$ medium change), which contained high levels of BDNF. Cells that were first cultivated in doxycycline-containing medium and changed to doxycycline-free medium at time 0 (red line) nearly completely shut down gene expression after $24 \mathrm{~h}$. Error bars in $\boldsymbol{A}$ and $\boldsymbol{B}$ are not always visible because of their small size.

and tet-on-GFP transduced fibroblasts were added and incubated overnight at $4^{\circ} \mathrm{C}$. The plates were washed and incubated with a chicken antiBDNF antibody overnight at $4^{\circ} \mathrm{C}(1: 2500$; Promega, Madison, WI), followed by five washes and overnight incubation with an anti-chicken IgY coupled to HRP (1:1000; Promega). After additional washes, $o$-phenylenediamine was used as substrate for signal detection. Absorbance in control wells coated with normal rabbit IgG were subtracted from wells coated with anti-BDNF IgG to correct for nonspecific binding. Recombinant human BDNF (Peprotech, Rocky Hill, NJ) was used as ELISA standard.

\section{Animal subjects}

A total of 128 adult female Fischer 344 rats weighing 150-200 g (3-4 months old) were subjects in this study. Animals were housed in standard laboratory cages and were provided access to food and water ad libitum. Rats were maintained under $12 \mathrm{~h}$ light/dark cycles. National Institutes of used for ELISA analysis received no graft (see below). For postoperative care, animals received $3 \mathrm{ml}$ of Ringer's lactate subcutaneously twice daily for $3 \mathrm{~d}$, manual bladder evacuation for $10 \mathrm{~d}$, and $25 \mathrm{mg}$ ampicillin twice daily for 2 weeks.

Animals were either continuously treated with doxycycline (D-9891; Sigma $)$ in the drinking water $(1 \mathrm{mg} / \mathrm{ml}$ in $5 \%$ sucrose $)$ or received doxycycline for the time indicated (Fig. $1 B$ ), followed by withdrawal of doxycycline, or animals received no doxycycline (5\% sucrose only) for the entire experimental period. Figure $1 B$ summarizes the treatment and survival of animals and the number of animals for histological analysis and quantification.

\section{Tissue processing}

Animals were transcardially perfused with $100 \mathrm{ml}$ of cold $0.1 \mathrm{M}$ PBS, followed by $300 \mathrm{ml}$ of $4 \%$ paraformaldehyde in PBS. Spinal cords were removed, postfixed overnight, and cryoprotected in 30\% sucrose. Sagittal sections were cut on a cryostat set at $35 \mu \mathrm{m}$ intervals. Every seventh section was mounted on glass slides for Nissl staining, and remaining sections were processed for immunolabeling. All sections were processed free floating, and endogenous peroxidase activity was blocked with $0.6 \%$ hydrogen peroxide in TBS for $15 \mathrm{~min}$. Sections were blocked with 5\% horse serum (for monoclonal antibodies) or 5\% goat serum (for polyclonal antibodies) for $1 \mathrm{~h}$ at room temperature. Sections were incubated overnight at $4{ }^{\circ} \mathrm{C}$ with the following primary antibodies: mouse antineurofilament (NF) (NF-200; 1:4000; Chemicon, Temecula, CA), mouse anti-p75 (1:250; 192MB hybridoma supernatants), mouse anti-P0, specific for peripheral myelin (1:4000; gift from Dr. J. Archelos, University of Graz, Graz, Austria) (Archelos et al., 1993).

After washing in Tris-buffered saline, sections were incubated with biotinylated secondary antibodies (1:200; Vector Laboratories, Burlingame, CA) for $1 \mathrm{~h}$ at room temperature, followed by $1 \mathrm{~h}$ incubation in avidin-biotinylated peroxidase complex (Elite kit; 1:100; Vector Laboratories) at room temperature. Diaminobenzidine $(0.05 \%)$ with nickel chloride $(0.04 \%)$ were used as chromagens, with reactions sustained for 0.5-10 min at room temperature. The sections were then mounted on gelatin-coated slides, dehydrated, and coverslipped. Photographs were taken using a Spot digital camera connected to an Olympus Optical (Melville, NY) AX-70 microscope.

For fluorescent labeling of sections, nonspecific labeling was blocked in TBS with $0.25 \%$ Triton X-100 (TBST) and 5\% donkey serum, and sections were incubated in primary antibodies diluted in TBST with $1 \%$ donkey serum at $4^{\circ} \mathrm{C}$ overnight at the following dilutions: goat anti-GFP (1:1500; Rockland Immunochemicals, Gilbertsville, PA), rabbit anti-S100 (1:2000; DakoCytomation Carpinteria, CA), rabbit antisynaptophysin (1:500; Chemicon), rabbit anti-microtubule-associated protein 2 (MAP2) (1:500; Chemicon), and mouse anti-GFAP (1:1500; 

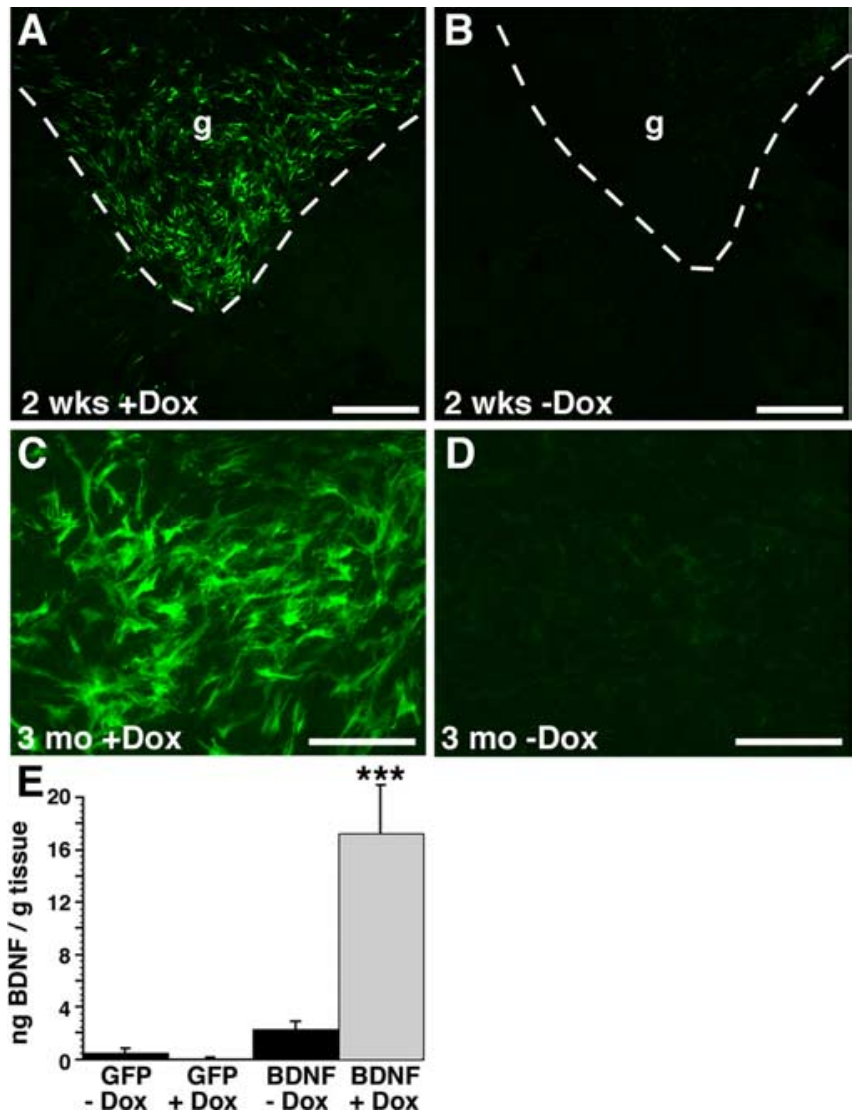

Figure 4. Regulated expression of GFP and BDNF in vivo. $A, B$, GFP fluorescence in grafts of genetically modified primary fibroblasts in the injured spinal cord 2 weeks after grafting. $\boldsymbol{A}$, Animals that were treated with doxycycline (+Dox) show GFP fluorescence in the graft, whereas no GFP fluorescence can be found in animals that received GFP grafts in the absence of doxycycline administration (-Dox) (B). Host/graft (g) interface is indicated by dashed lines. $\boldsymbol{C}$, $D$, Immunolabeling for GFP shows expression in grafts of animals that were treated with doxycycline for 3 months $(\boldsymbol{C})$, whereas very weak GFP expression can be found in animals that received GFP grafts in the absence of doxycycline administration (-Dox) for 3 months (D). $\boldsymbol{E}$, Regulated expression of BDNF in primary fibroblasts grafted to the injured spinal cord. Two weeks after grafting, GFP- and BDNF-expressing grafts were dissected from animals that were either untreated $(-D o x)$ or treated with doxycycline in the drinking water ( + Dox) to turn gene expression on. BDNF levels were determined by ELISA. tet-on-BDNF transfected fibroblast grafts from animals that were treated with doxycycline (BDNF + Dox) show significantly higher BDNF levels than untreated animals (BDNF - Dox) and animals with control grafts $\left({ }^{* * *} p<\right.$ 0.001). BDNF levels in untreated animals with tet-on-BDNF transfected fibroblasts grafts are not significantly different from treated ( $p=0.76$ ) or untreated ( $p=0.85$ ) GFP transfected control animals. Scale bar: $\boldsymbol{A}, \boldsymbol{B}, 283 \mu \mathrm{m} ; \boldsymbol{C}, \boldsymbol{D}, 138 \mu \mathrm{m}$.

Chemicon). After washing, sections labeled for GFP were incubated for $2.5 \mathrm{~h}$ at room temperature with donkey anti-goat antibodies conjugated to Alexa-488 (1:200; Invitrogen, Carlsbad, CA). For double labelings, sections were incubated simultaneously with rabbit and mouse primary antibodies overnight at the dilutions indicated above (S-100/GFAP, MAP2/GFAP, and synaptophysin/GFAP). Sections were washed and incubated with donkey anti-mouse and donkey anti-rabbit antibodies conjugated to Alexa-488 and Alexa-594 (1:200), respectively.

\section{Quantification of axon and Schwann cell density}

Axon density in NF-labeled sections and density of myelinating Schwann cells in P0-labeled sections within grafts was measured using NIH Image software on video images of tissue sections transmitted by a Sony (Tokyo, Japan) CCD digital camera, as described previously (Blesch et al., 1999, 2004; Blesch and Tuszynski, 2003). Briefly, for each axonal label, two to three sections per animal were selected that contained the largest volume of graft. The number of pixels occupied by immunolabeled axons or Schwann cells within a fixed box size of $640 \times 480$ pixels placed in the
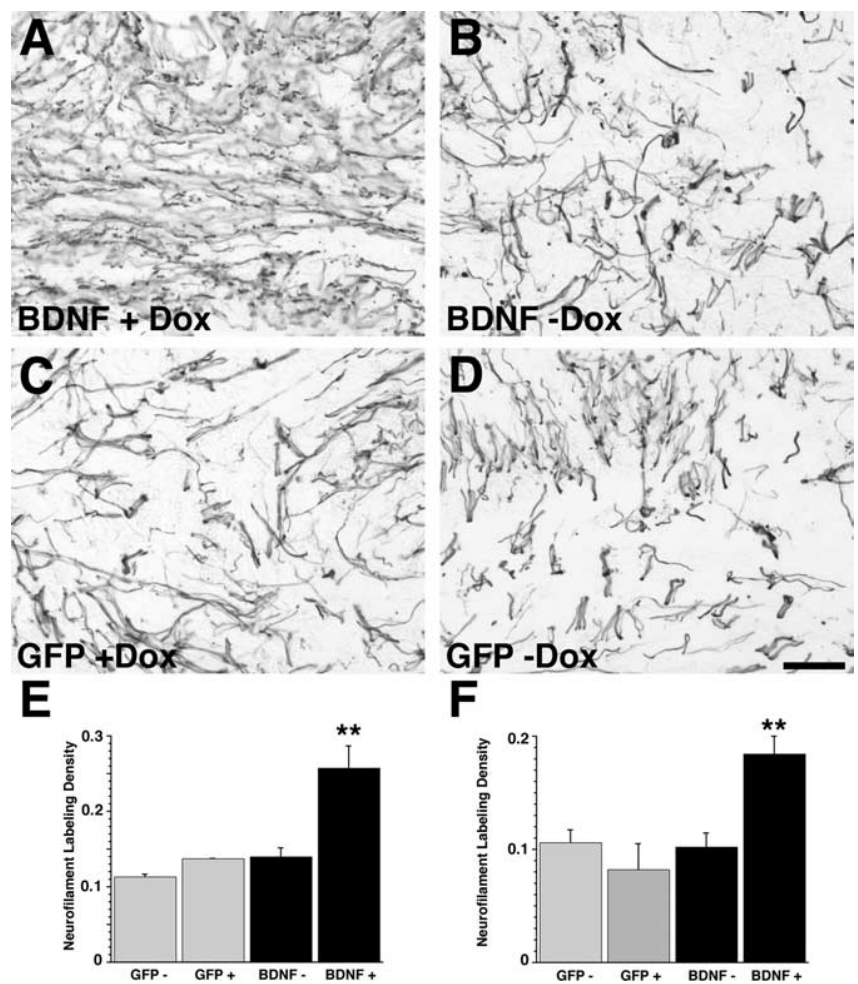

$\mathbf{F}$

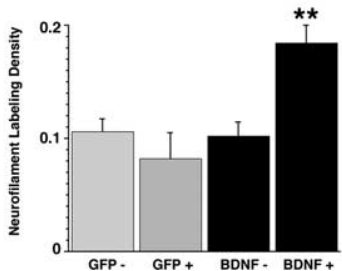

Figure 5. Axonal growth in response to regulated BDNF expression in the injured spinal cord. $\boldsymbol{A}$, Two weeks after grafting, BDNF-secreting grafts of animals that received doxycycline in the drinking water are densely penetrated by neurofilament-labeled axons. $\boldsymbol{B}$, BDNF-secreting grafts in animals that did not receive doxycycline (BDNF is turned off) exhibit very few axons and do not differ from GFP-expressing grafts of animals that received doxycycline to turn gene expression on $(\boldsymbol{C})$ or received no doxycycline $(\boldsymbol{D})$. $\boldsymbol{E}, \boldsymbol{F}$, Quantification of NF labeling at 2 weeks $(\boldsymbol{E})$ and 6 weeks $(\boldsymbol{F})$ shows significant higher labeling density in animals grafted with BDNFsecreting fibroblasts if gene expression was turned on continuously for 2 and 6 weeks, respectively (BDNF + Dox). Animals with BDNF grafts that received no doxycycline (BDNF - Dox; gene expression turned off) are not significantly different from control animals with GFPexpressing fibroblasts grafts (GFP + , GFP-; ${ }^{* *} p<0.01$ comparing BDNF + with other groups). Scale bar, $67 \mu \mathrm{m}$.

epicenter of the graft (identified by phase-contrast microscopy and serial Nissl-labeled sections) was measured at $100 \times$ magnification $(10 \times$ objective) in sections immunolabeled for NF, p75, and P0, respectively. Thresholding values on video images were chosen such that only immunolabeled axons or Schwann cells were measured, and light nonspecific background labeling was not detected. Nonspecific staining or artifactual spots were edited from images. Total labeled pixels were divided by the sample box size $(640 \times 480$ pixels $)$ to obtain mean axon and Schwann cell density per area.

For quantification of S-100 labeling density, sections were double labeled for S-100 and GFAP to discriminate S-100 labeling of astrocytes and Schwann cells. Grayscale photographs were taken at $100 \times$ magnification using epifluorescent illumination and appropriate wavelength filters. Grafts devoid of GFAP labeling were outlined using NIH ImageJ in photographs of GFAP labeling, and S-100 labeling density was quantified in the previously outlined GFAP-negative area as described above.

In vivo BDNF expression

In vivo expression of BDNF was examined in 54 animals using a BDNFspecific ELISA. Animals were grafted with tet-on-BDNF transduced fibroblasts $(n=24)$ and were either untreated $(n=11)$ or treated with doxycycline in the drinking water $(n=12)$. Additionally, five animals were treated for 3 weeks with doxycycline, followed by 1 week without doxycycline administration. Nine animals grafted with tet-on-GFP transduced fibroblasts were untreated, and eight animals were treated with doxycycline. In addition four naive, uninjured animals and four animals that received a dorsal hemisection but no graft were analyzed. 

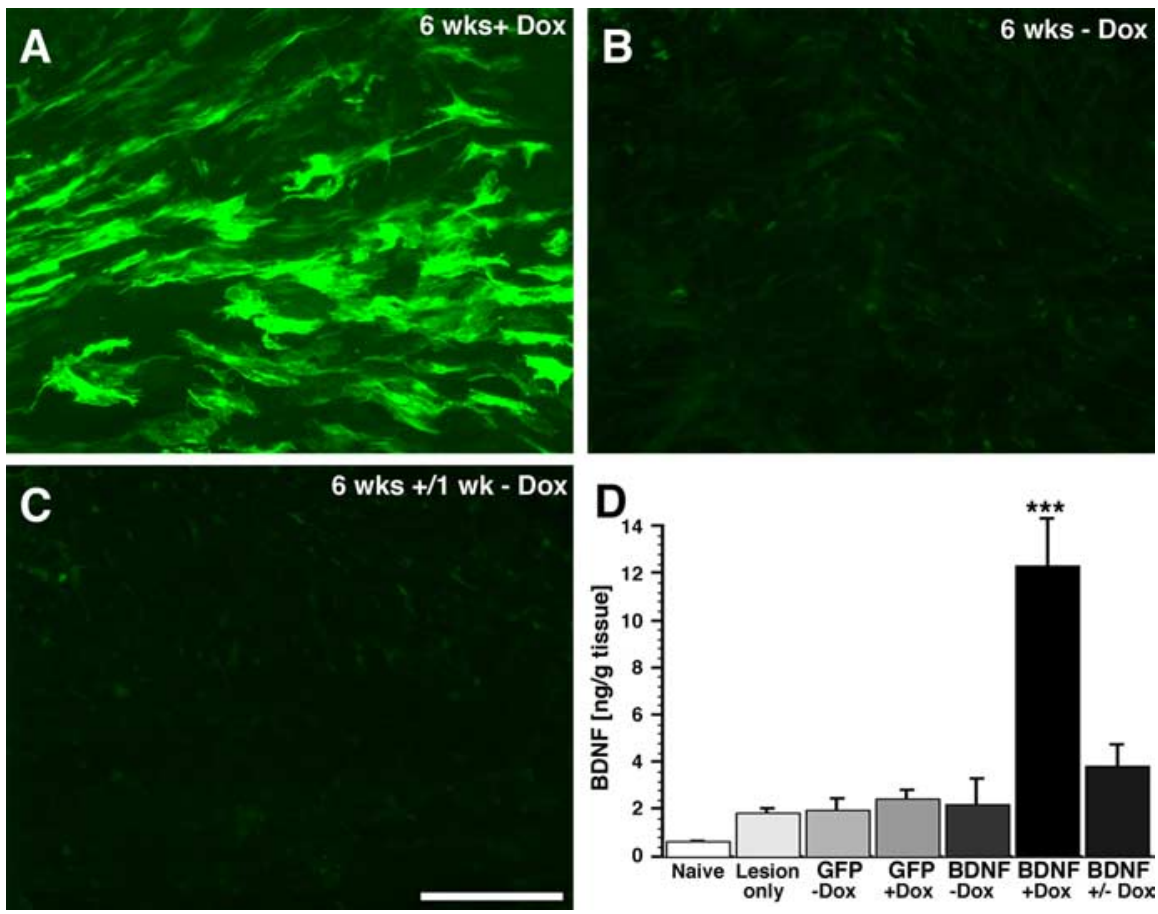

Figure 6. GFP and BDNF gene expression can be turned off within 1 week after doxycycline removal. $\boldsymbol{A}-\boldsymbol{C}$, Animals that received GFP grafts and were treated with doxycycline for 6 weeks show strong GFP expression $(\boldsymbol{A})$, whereas very weak GFP expression can be found in animals that received GFP grafts in the absence of doxycycline administration (-Dox) for 6 weeks $(\boldsymbol{B})$. C, Animals that received GFP grafts and were first treated with doxycycline for 6 weeks and then received no doxycycline for 1 week to turn gene expression off show also no GFP expression. D, BDNF expression levels in intact spinal cord (naive), animals that received no graft (lesion only), and BDNF- and GFP-expressing grafts in animals that were either untreated (-Dox) for 3 weeks, treated with doxycycline (+Dox) for 3 weeks to turn gene expression on, or treated with doxycycline for 3 weeks followed by withdrawal of doxycycline for 1 week (+/-Dox). tet-on-BDNF transfected fibroblast grafts from animals that were treated with doxycycline (BDNF + Dox) show significant higher BDNF levels than untreated animals (BDNF - Dox), animals with control grafts $(G F P+$ and GFP - ), and animals that were first treated with doxycycline followed by doxycycline removal (BDNF + / - Dox). BDNF levels in untreated animals with tet-on-BDNF transfected fibroblasts grafts (BDNF - Dox) or animals that were treated with doxycycline for 3 weeks followed by removal of doxycycline for 1 week are not significantly different from treated or untreated GFP transfected control animals or animals that received no graft. Scale bar: $\boldsymbol{A}-\boldsymbol{C}, 138 \mu \mathrm{m}$. ${ }^{* *} p \leq 0.0001$ comparing BDNF + Dox with other groups.

Two, 3, or 4 weeks after grafting (Fig. $1 B$ ), animals received an overdose of anesthesia and were transcardially perfused with $50 \mathrm{ml}$ of ice-cold saline. The graft was identified by a slight but distinct color difference from the surrounding host tissue using an operating microscope, and fresh tissue was obtained from the graft site and dissected from the surrounding host tissue. In naive animals, the T7/8 spinal cord was sampled, and, in lesioned animals that received no graft, the lesion site and tissue immediately surrounding the lesion was analyzed. Samples were immediately frozen on dry ice until additional processing. Sample lysates were obtained by sonication in lysate buffer (PBS with $0.25 \%$ Triton X-100, 5 mM EDTA, 0.5\% BSA, $1 \mathrm{~mm}$ PMSF, and $1 \mu \mathrm{l} / \mathrm{ml}$ aprotinin), and serial dilutions were processed in a BDNF ELISA as described above.

\section{Statistics}

Data are presented as mean \pm SEM. Two-way comparisons were tested using two-tailed Student's $t$ tests. Multiple group comparisons were made by ANOVA, followed by post hoc Fisher's tests using Statview software (SAS Institute, Cary, NC). A significance criterion of 0.05 was used in all analyses. Observers were blinded to experimental group identity in all analyses.

\section{Results}

In vitro characterization of tet-on-BDNF and tet-on-GFP transduced primary fibroblasts

Primary rat fibroblasts genetically modified with retroviruses for the controlled expression of the reporter gene GFP were selected for neomycin resistance and exposed to different doses of doxycycline in vitro. Examination of cells under fluorescent illumination showed a dose-dependent induction of fluorescence in vitro (Fig. 2). Because of the long half-life of GFP and the difficulty to measure GFP expression quantitatively, a more detailed analysis was performed for inducible BDNF expression.

Regulation of BDNF production in vitro was evaluated by transduction of three primary fibroblast cell lines and selection for neomycin resistance. Cells cultivated without doxycycline produced $0.5-1 \mathrm{ng} \mathrm{BDNF} / 10^{6}$ cells per $24 \mathrm{~h}$. After addition of $1 \mu \mathrm{g} / \mathrm{ml}$ doxycycline, BDNF expression increased to 77-307 ng BDNF/ $10^{6}$ cells per $24 \mathrm{~h}$. Thus, BDNF expression could be regulated on average 187-fold and ranged from 96- to 300-fold (Table 1).

The initial characterization of regulatable expression of BDNF and GFP was done in the presence or absence of 1 $\mu \mathrm{g} / \mathrm{ml}$ doxycycline. However, substantially lower levels of doxycycline could be capable of turning on BDNF expression, and ideally various levels of BDNF expression should be obtainable depending on the amount of doxycycline present. Analysis of the dose-response curve of tet-on-BDNF transfected cells exposed to various doses of doxycycline demonstrated that concentrations of doxycycline as low as $1 \mathrm{ng} / \mathrm{ml}$ induced expression and secretion of BDNF into the cell culture medium (Fig. $3 A$ ). The $\mathrm{ED}_{50}$ lies in the range of $20-30 \mathrm{ng} / \mathrm{ml}$ doxycycline. Thus, expression of BDNF in these cells is dependent on the concentration of doxycycline in the medium and can be accordingly modulated.

The time course of BDNF expression was measured in the presence or absence of doxycycline (Fig. $3 B$ ). At time 0 , doxycycline $(1 \mu \mathrm{g} / \mathrm{ml})$ was added to the medium of cells previously grown in antibiotic-free medium. In parallel cultures, doxycycline was withdrawn from cells previously grown with doxycycline. As shown in Figure 3B, BDNF secretion was rapidly induced within $12 \mathrm{~h}$ of exposure to doxycycline, and the medium harvested at the $24 \mathrm{~h}$ time point (containing the protein secreted between 12 and $24 \mathrm{~h}$ ) contained maximum levels of BDNF. In contrast, withdrawal of doxycycline resulted in a rapid decline in BDNF expression within $12 \mathrm{~h}$ after doxycycline withdrawal, and the medium harvested at the $24 \mathrm{~h}$ time point (containing the

Table 1. In vitro BDNF production of three independent primary fibroblast cell lines (ng/10 ${ }^{6}$ cells per $24 \mathrm{~h}$ )

\begin{tabular}{llll}
\hline & \multicolumn{3}{l}{ BDNF production $\left(\mathrm{ng} / 10^{6}\right.$ cells per $24 \mathrm{~h}$} \\
\cline { 2 - 4 } Cell line & - Dox & + Dox & Fold regulation \\
\hline FF9021 & 1.03 & 307.61 & 299 \\
FF501 & 0.47 & 77.33 & 165 \\
FF9022 & 1.00 & 95.60 & 96 \\
\hline
\end{tabular}


protein secreted between 12 and $24 \mathrm{~h}$ ) contained virtually no BDNF protein. Parallel cultures that were grown with constant levels of antibiotic continued to express BDNF. In contrast, cultures grown without antibiotic exhibited substantially reduced $\mathrm{BDNF}$ expression close to the detection limit of the ELISA.

\section{Regulated expression of BDNF and GFP in vivo}

To determine whether gene expression can also be regulated in vivo, fibroblasts genetically modified to express GFP under the control of the tetracycline-inducible promoter were grafted to the rat spinal cord after a dorsal hemisection at midthoracic level. Two weeks after grafting, GFP fluorescence was only visible in animals that continuously received doxycycline in the drinking water to turn gene expression on. No green fluorescence was detectable in animals that received no doxycycline (Fig. 4). The same extent of regulation could be observed 3 months after grafting with or without continuous doxycycline treatment.

To measure the degree of regulation quantitatively, we determined expression levels of BDNF in vivo. (Fig. 4E) Two weeks after grafting, fibroblast grafts of animals that received tet-on-BDNF transduced fibroblasts and were either continuously treated with doxycycline or untreated were dissected, and BDNF protein levels were determined by ELISA. To measure baseline levels of BDNF present at the injury site, BDNF expression levels in animals grafted with tet-on-GFP transduced fibroblasts were also analyzed. BDNF protein levels were highest in grafts of animals with tet-on-BDNF transduced fibroblasts that received doxycycline in the drinking water and were significantly different from all other groups $(p<0.001)$. BDNF expression in grafts of genetically modified fibroblasts harboring a tet-on-BDNF vector was $\sim 10$-fold lower if animals were not treated with doxycycline and did not significantly differ from levels of BDNF in control animals with GFP-expressing grafts. Thus, significant amounts of GFP and $\mathrm{BDNF}$ are only expressed in the presence of doxycycline.

\section{Regulation of axonal growth in vivo}

Next, we investigated whether axonal growth can be modified in response to regulated BDNF expression. Animals received grafts of syngenic fibroblasts expressing GFP or BDNF under the control of the tetracycline-inducible promoter. Two weeks later, controlled expression of BDNF significantly enhanced differential axonal growth into grafts of genetically modified primary fibroblasts (Fig. 5). Grafts of BDNF-secreting cells in animals that received no doxycycline in the drinking water (gene expression turned off) showed a similar extent of axonal penetration as con-
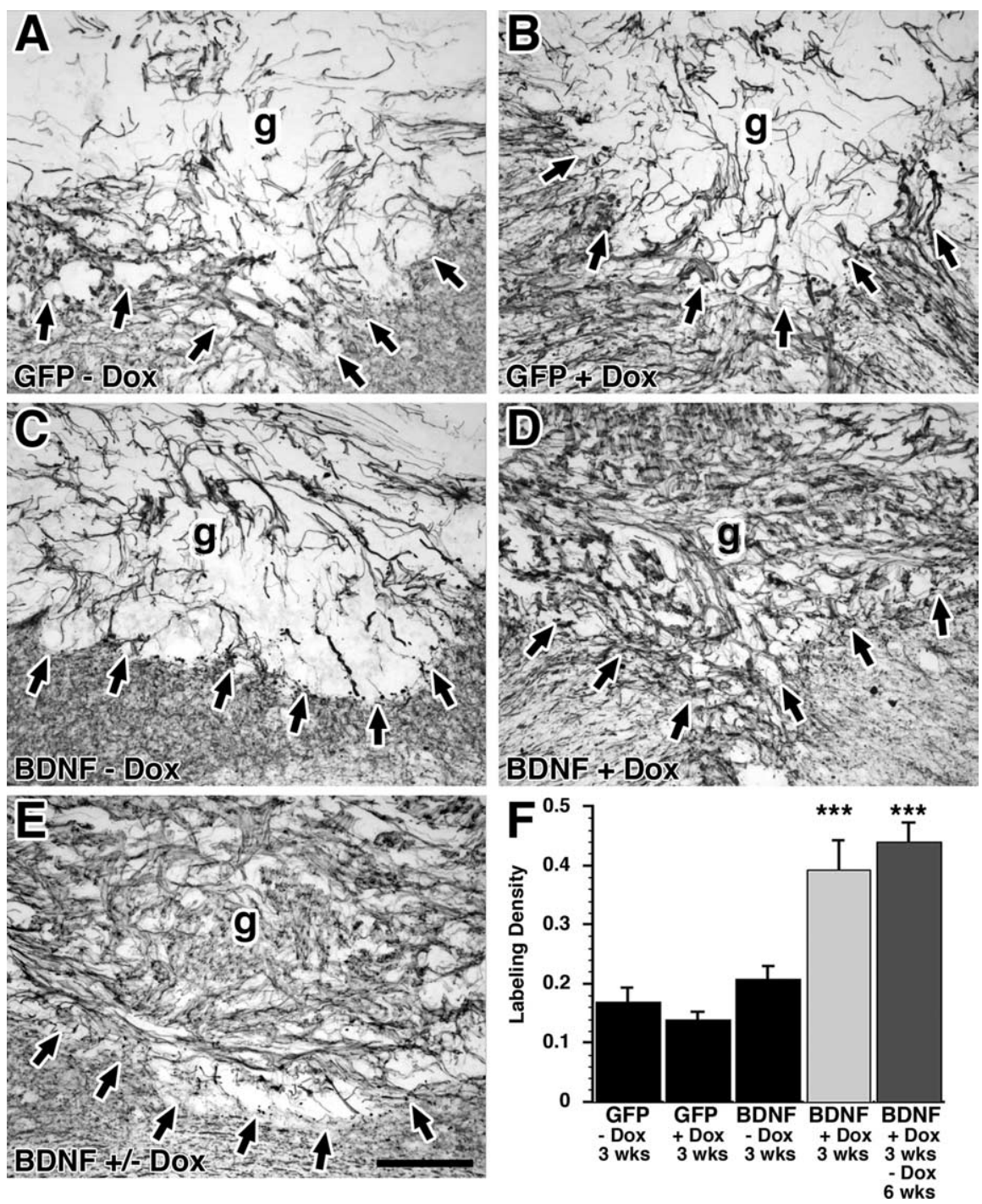

Figure 7. Axons remain within BDNF-expressing grafts after gene expression has been turned off. $\boldsymbol{A}$-C, GFP-expressing grafts in animals that did not receive doxycycline (GFP - Dox) $(\boldsymbol{A})$ or received doxycycline (GFP + Dox) for 3 weeks to turn gene expression on $(\boldsymbol{B})$ exhibit very few axons and do not differ from BDNF-expressing grafts in animals that received no doxycycline (BDNF - Dox) (C). D, BDNF-secreting grafts in animals that received doxycycline for 3 weeks (BDNF + Dox) are densely penetrated by neurofilament-labeled axons, and axonal density is similar in BDNF-secreting grafts in animals that were first treated with doxycycline for 3 weeks followed by removal of doxycycline for 6 weeks to turn gene expression off (BDNF +/ - Dox) (E). $\boldsymbol{F}$ Quantification of axonal density shows significantly higher labeling density in doxycycline-treated animals with BDNF-secreting grafts compared with doxycycline-treated or untreated animals with GFP-expressing grafts or animals with BDNF-secreting grafts that were untreated. Reduction of BDNF expression after 3 weeks by withdrawal of doxycycline for 6 weeks does not result in a significant reduction of axonal density. Scale bar, $170 \mu \mathrm{m}$. ${ }^{* *} p<0.001$. Arrows indicate host/graft $(\mathrm{g})$ interface.

trol GFP-expressing grafts after 2 weeks. In contrast, BDNF coding fibroblast grafts in animals that were continuously treated with doxycycline to turn gene expression on were densely penetrated by neurofilament-labeled axons after 2 weeks $(p<0.01$ compared with GFP and no doxycycline groups). This differential axonal growth response persisted 6 weeks after grafting and continuous doxycycline treatment (Fig. $5 F)(p<0.01)$. Similar results were obtained using immunolabeling for the low-affinity neurotrophin receptor p75 (data not shown).

Thus, BDNF and GFP expression can be regulated in genetically modified fibroblast grafts in the injured spinal cord for at least 6 weeks and 3 months, respectively. Animals containing grafts of BDNF-secreting cells that were not turned on showed no difference from GFP grafts in in vivo levels of BDNF in grafts and 

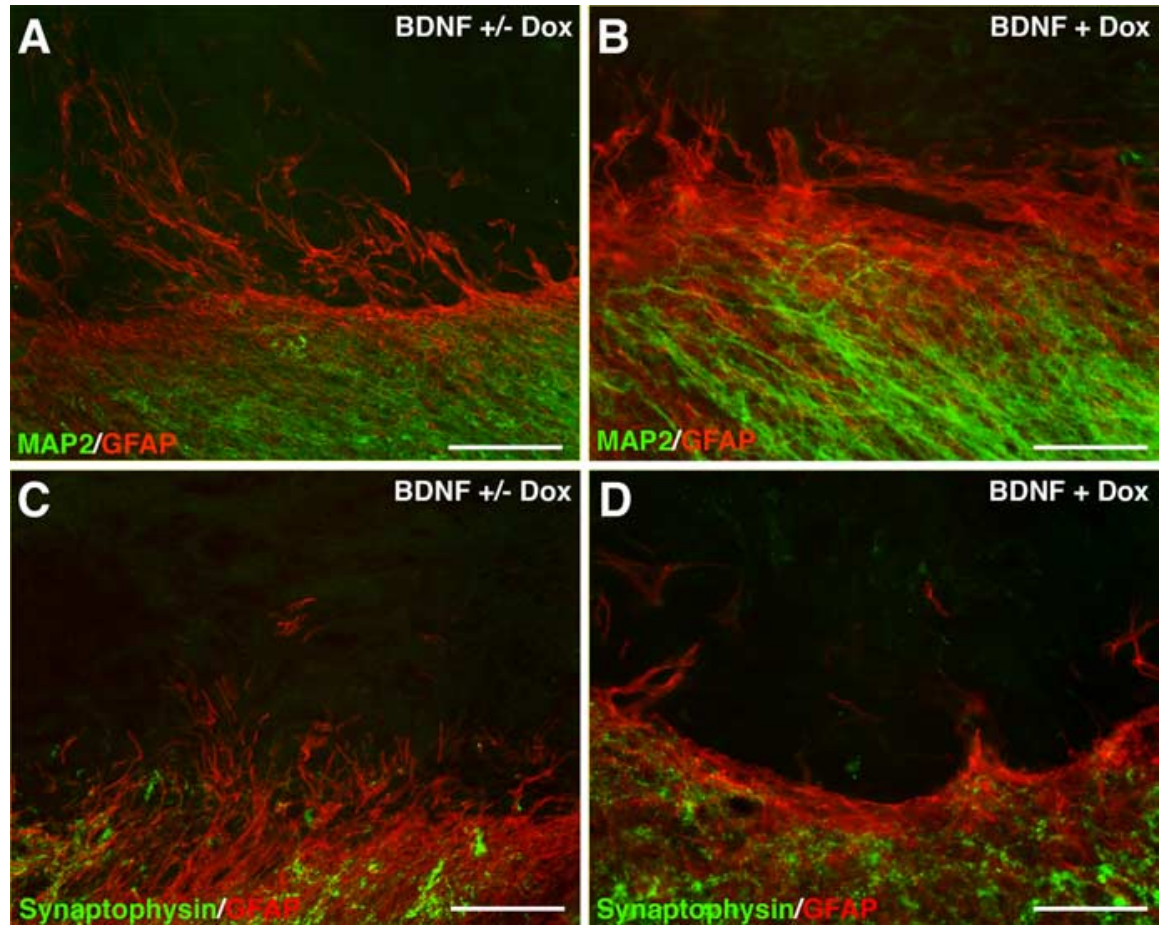

Figure 8. Axons do not form synapses in BDNF-expressing grafts. $A, B$, Double labeling for GFAP (red) to identify the host/graft interface and MAP2 (green) to identify dendrites indicates a slight retraction of dendrites from the host/graft interface. This is equally observed in animals that were first treated for 3 weeks with doxycycline to turn gene expression on followed by doxycycline withdrawal for 6 weeks to turn gene expression off $(\boldsymbol{A})$ and in BDNF-expressing grafts 3 weeks after grafting (BDNF + Dox; turned on for 3 weeks) ( $\boldsymbol{B})$. C, D, Analogous to MAP2 labeling, synaptophysin immunolabeling is restricted to the host spinal cord and is only occasionally found in the graft in close proximity to the host/graft interface. No differences are observed comparing grafts of animals with BDNF-expressing grafts 3 weeks after grafting (BDNF + Dox; turned on for 3 weeks) $(\boldsymbol{C})$ and grafts of animals with transient BDNF expression (BDNF + / - Dox; BDNF turned on for 3 weeks by doxycycline administration followed by doxycycline withdrawal for 6 weeks to turn gene expression off) (D). Scale bars: $\boldsymbol{A}, 170 \mu \mathrm{m} ; \boldsymbol{B}, \boldsymbol{C}, 85 \mu \mathrm{m} ; \boldsymbol{D}, 106 \mu \mathrm{m}$.

no augmentation of axonal growth. Administration of doxycycline turned gene expression on in cell grafts to levels sufficiently high to induce significantly augmented axon growth.

\section{Expression of GFP and BDNF can be discontinued in vivo}

To evaluate whether gene expression can be turned off by withdrawal of doxycycline from the drinking water, animals grafted with tet-on-GFP-expressing fibroblasts were treated for 6 weeks with doxycycline, followed by withdrawal of doxycycline for 1 week. Control animals were either continuously treated with doxycycline in the drinking water (gene expression turned on) or received no doxycycline (gene expression turned off). We found that animals that received doxycycline for 6 weeks to turn gene expression on, and subsequently received no doxycycline for 1 week, showed no or very weak GFP expression and did not differ from animals that never received doxycycline (Fig. 6). As expected, animals that were continuously doxycycline treated showed extensive GFP expression.

Similar results were obtained by measuring levels of BDNF by ELISA in grafts dissected from animals with regulated GFP and BDNF expression (Fig. 6D). Three weeks after grafting, highest levels of BDNF were detected in animals that continuously received doxycycline in the drinking water (gene expression turned on) and differed significantly from all other groups $(p<0.0001)$. Animals with GFP-expressing grafts did not differ from animals that received regulatable BDNF-expressing grafts without doxycycline treatment (gene expression turned off). Importantly, animals that were treated for 3 weeks with doxycycline, followed by
1 week without doxycycline treatment, showed reductions in BDNF to levels that were not significantly different from control animals containing GFP-expressing grafts, from animals with BDNFexpressing grafts that were never treated with doxycycline (never turned on), or from animals that received no graft. BDNF levels in injured animals without grafts were approximately threefold higher at the lesion site than in the normal spinal cord $(p<0.01$, Student's $t$ test $)$. Thus, BDNF levels revert within 1 week from supraphysiological to physiological levels on doxycycline withdrawal.

\section{Axonal penetration is sustained when BDNF expression is turned off}

To determine whether withdrawal of doxycycline and subsequent reduction in BDNF expression would result in axonal withdrawal, axonal penetration into BDNF- and GFP-expressing grafts in animals that received doxycycline transiently in the drinking water was analyzed quantitatively (Fig. 7). Similar to the previous experiment, significant axonal growth into the graft was only observed when BDNF expression was turned on by doxycycline administration for 3 weeks. Control animals with tetracycline-regulatable GFPexpressing grafts that were either doxycycline treated for 3 weeks (GFP turned on) or untreated (GFP turned off) were not significantly different from animals that received regulatable BDNF-secreting grafts without doxycycline treatment (BDNF turned off). Most importantly, no significant loss of axonal density was observed when BDNF expression was first turned on for 3 weeks and subsequently turned off for 6 weeks by doxycycline withdrawal (Fig. 7E). Thus, axonal density did not decrease after BDNF expression was turned off for 6 weeks, and host axons persisted in the lesion site.

\section{Grafts transiently expressing BDNF are devoid of synapses but contain Schwann cells}

To determine whether axonal penetration might have been sustained by formation of synapses in grafts, sections were examined by immunohistochemistry for the presence of MAP2-labeled dendrites and synaptophysin-labeled synapses. No MAP2 labeling was present in any BDNF- or GFP-expressing graft (Fig. $8 A, B)$. Consistent with this finding, synaptophysin labeling was restricted to host gray matter and to the immediate vicinity of the host/graft interface (Fig. 8C,D).

Schwann cells identified by S-100 and myelinating Schwann cells identified by P0 labeling (Archelos et al., 1993) were present within grafts, as reported previously (Figs. 9, 10) (Blesch et al., 2004). The density of Schwann cells found in grafts after 3 weeks of BDNF expression (with doxycycline) was significantly higher compared with GFP control grafts or BDNF grafts lacking in vivo BDNF expression (without doxycycline treatment). If BDNF expression was turned on for 3 weeks and subsequently turned off for 6 weeks by doxycycline removal, Schwann cell density and myelination did not change and remained at levels that were 
significantly different from GFP control grafts or BDNF grafts lacking in vivo BDNF expression.

\section{Discussion}

Findings of this study indicate that transient growth factor delivery is sufficient to sustain long-term axonal projection into a site of SCI, in the absence of axonal target reinnervation and synapse formation. Using a recently modified tetracyclinedependent system for regulating gene expression in a "positive" manner, gene expression was induced by oral administration of doxycycline in the drinking water. BDNF protein levels could be positively regulated over a range of 100- to 300 -fold in vitro and from physiological levels $(1-2 \mathrm{ng} / \mathrm{g})$ in the off state to an average of $12 \mathrm{ng} / \mathrm{g}$ after oral doses of doxycycline. In the on state, axons robustly penetrated the BDNF-rich lesion site, in which synapses did not form. Importantly, when BDNF was turned on in the lesion site for 3 weeks and then turned off for another 6 weeks, axon density in the graft remained high and did not differ from animals with continuous BDNF administration for a 3 week postlesion period. Thus, levels of BDNF can be positively regulated in vivo with an orally administered agent. Moreover, the ability to regulate BDNF in vivo reveals that a continued stimulus for growth, or synapse formation, is not required to maintain a novel topography of axons after injury.

The ability to sustain a nonfunctional axon or axon branch in the absence of a synapse or an ongoing trophic stimulus in the injured CNS appears to diverge from observations in the developing nervous system. During development, neural activity strengthens nascent projections, whereas synaptically inactive projections are pruned. However, axons are sustained in the injured CNS in our model in the absence of a known target despite downregulation of growth factor expression to levels indistinguishable from animals lacking BDNF-secreting cells.

Several potential mechanisms could underlie this observation. One source of axon-sustaining support in the lesion/graft site might be Schwann cells migrating into grafts from adjacent nerve roots. Schwann cells produce low levels of neurotrophic factors, including NGF, BDNF, and NT-3 (Heumann et al., 1987; Funakoshi et al., 1993; Naveilhan et al., 1997), extracellular matrix such as laminin, and cell adhesion molecules that could contribute to axonal persistence. Indeed BDNF amounts detected in vivo in control grafts of GFP-expressing cells and at the lesion site in animals without grafts might partially be derived from Schwann cells. Quantification of Schwann cell densities in the graft/lesion site indicated that expression of BDNF for 3 weeks resulted in an increased number of Schwann cells compared with control grafts.
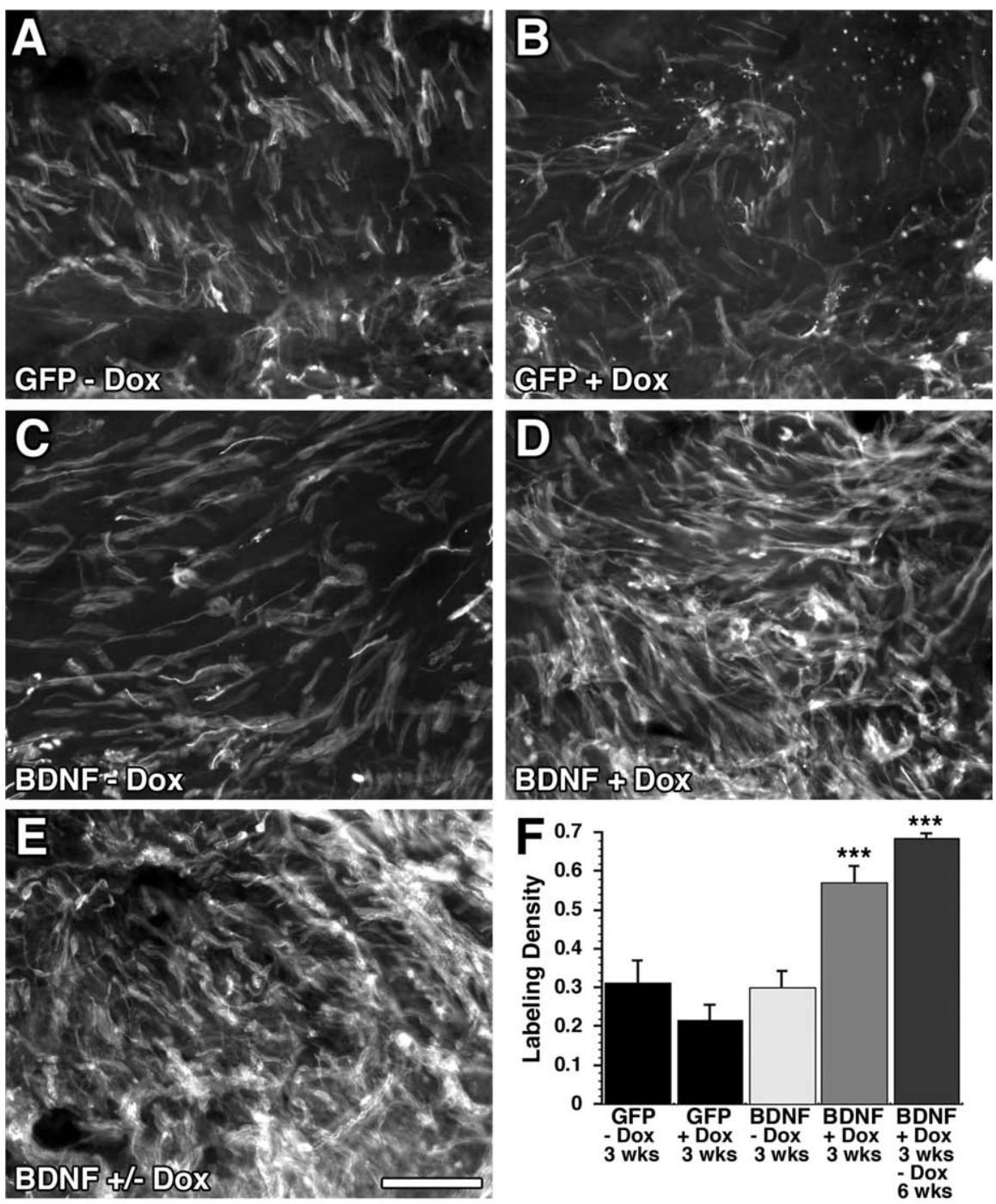

Figure 9. Schwann cell numbers are increased by BDNF delivery and remain high after BDNF expression is turned off. $\boldsymbol{A}-\boldsymbol{C}$ $S$-100 labeling density in GFP-expressing control grafts from untreated (GFP - Dox) (A) and doxycycline-treated animals (GFP + Dox) $(\boldsymbol{B})$ does not differ from grafts of untreated animals with BDNF expressing grafts (BDNF - Dox; gene expression turned off)

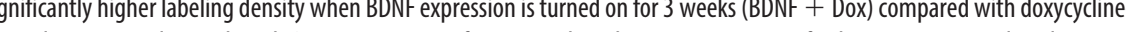
reated or untreated animals with GFP-expressing grafts or animals with BDNF-secreting grafts that were untreated. Reduction of BDNF expression after 3 weeks by withdrawal of doxycycline for 6 weeks does not result in a reduction of Schwann cell density $\left({ }^{* * *} p<0.001\right.$ comparing BDNF + Dox or BDNF +/-Dox with GFP + Dox, GFP - Dox, or BDNF - Dox). Scale bar, $85 \mu \mathrm{m}$.

Turning off BDNF expression did not diminish the number of Schwann cells in a subsequent 6 week period. Thus, axon- and Schwann cell-derived soluble factors and direct contact might establish mutual signals that lead to a steady-state level of axon/ Schwann cell densities independent of external signals such as high neurotrophin expression. The close interaction between axons and Schwann cells within grafts, also reported in previous studies (Weidner et al., 1999; Blesch et al., 2004), is also reflected in the high density of Schwann cell myelination that remains high and does not diminish once BDNF expression is turned off.

Although axons did not form synapses within grafts, indicated by a lack of markers for dendrites and synapses, axonal bridging across the lesion/graft site could hypothetically result in novel connections beyond the lesion site that sustained axonal density in the graft. The lack of an axon growth stimulus beyond the 

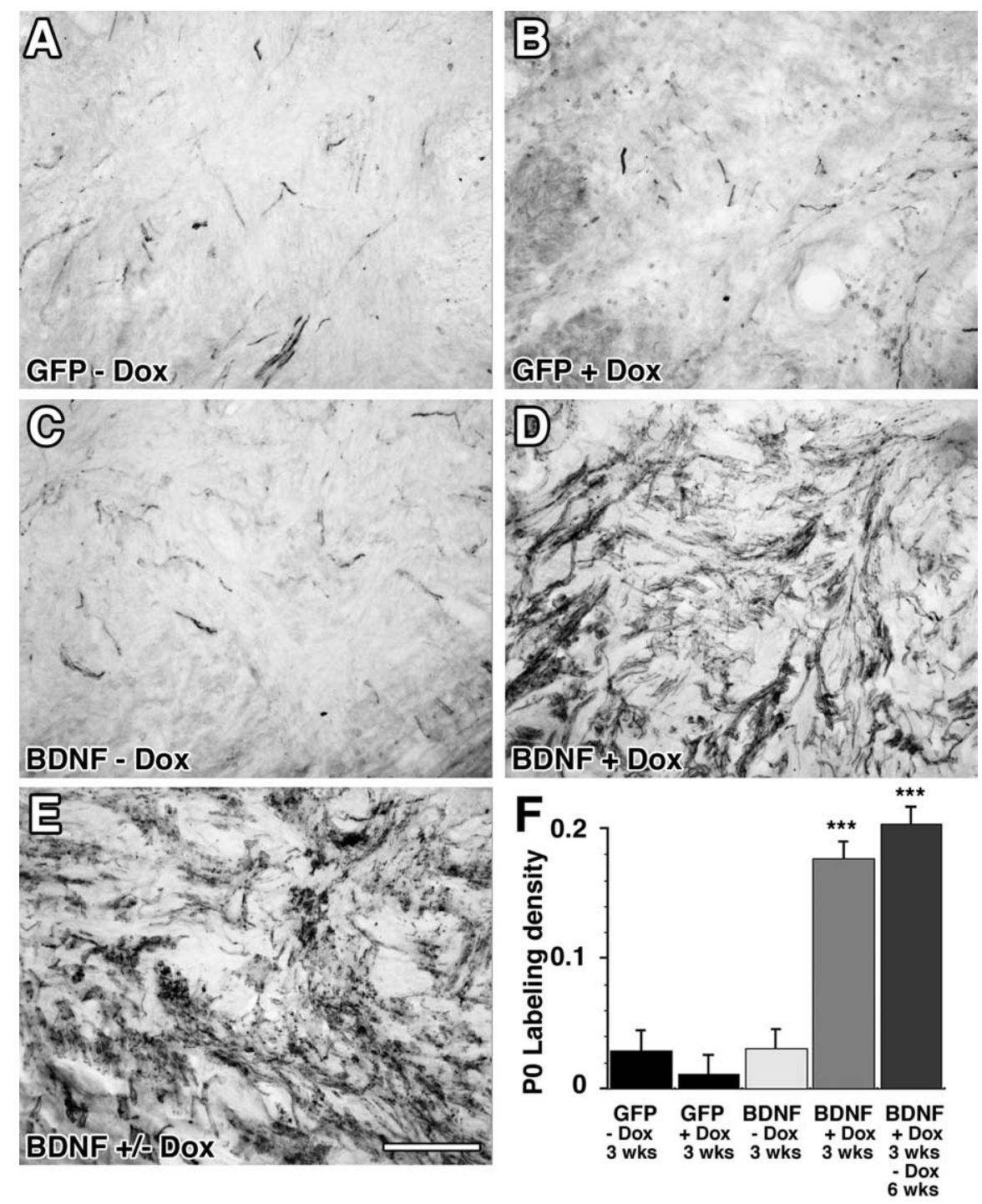

Figure 10. Schwann cell myelination is sustained after BDNF delivery is turned off. $\boldsymbol{A}-\boldsymbol{C}$, PO labeling indicates only very few myelinating Schwann cells in GFP-expressing control grafts of untreated (GFP - Dox) ( $\boldsymbol{A}$ ) or doxycycline-treated (GFP + Dox) (B) animals and in BDNF grafts of untreated animals (gene expression turned off; BDNF - Dox) (C). D, IfBDNF expression is turned on for 3 weeks (BDNF + Dox), the number of $P 0$-labeled Schwann cells is significantly increased. $\boldsymbol{E}$, Schwann cell density remains high if BDNF expression is turned on for 3 weeks and turned off in the subsequent 6 weeks. $F$, Quantification of P0 labeling density indicates a significant 15- to 20-fold increase in P0 labeling density if BDNF expression is turned on for 3 weeks (BDNF + Dox). This dense Schwann cell myelination is sustained in grafts of animals with transient BDNF expression (gene expression turned on for 3 weeks and turned off in the following 6 weeks (BDNF $+/-$ Dox; $^{* * *} p<0.0001$ comparing BDNF + Dox or BDNF + / - Dox with GFP + Dox, GFP - Dox, or BDNF - Dox). Scale bar, $136 \mu \mathrm{m}$.

lesion site and the presence of axonal growth inhibitors in the host spinal cord, including myelin-based inhibitors and inhibitory proteoglycans, renders this possibility unlikely. Indeed, previous studies have indicated that axons extending into grafts of growth factor-producing fibroblasts fail to bridge across the lesion/graft site and do not form functional distal connections (Blesch and Tuszynski, 2003; Blesch et al., 2004). Alternatively, axons within grafts after transient BDNF delivery could hypothetically be sustained by long-lasting modifications of collateral synaptic contacts in the surrounding host tissue. That is, collaterals of axons that penetrated the graft might have formed new connections in surrounding host tissue, or previously established connections may have been modified by BDNF, thereby sustaining axon branches in fibroblast grafts. However, there is no experimental evidence to support this mechanism, and an inactive axon collateral would hypothetically also be vulnerable to disuse-related degeneration. However, another potential mechanism through which axons in fibroblast grafts might be sustained over time despite downregulation of BDNF production is the production of other growth factors by fibroblasts. In such a case, fibroblastderived factors would have been insufficient to induce significant axonal growth initially but might have been sufficiently potent to sustain axons after they extended into grafts. This possibility cannot be excluded. Finally, one must entertain the possibility that axons, having regenerated into a target area as a result of tropic influences, persist in such a position unless actively perturbed or redirected. Supporting this possibility is the observation that transected axons in both the CNS and PNS are often detectable indefinitely adjacent to their transection sites. These axons have not reinnervated targets or formed synapses, yet many of these axons survive and persist adjacent to the lesion site for prolonged time periods of at least 1 year in animal models (Houle and Jin, 2001; Kwon et al., 2002).

We chose to use the tetracyclineinducible system (tet-on system) rather than the tetracycline repressible system (tet-off system) in this study because administration of doxycycline is only necessary during the period of active gene expression and not for extended periods thereafter. Our data demonstrate that expression of the neurotrophic factor BDNF and the reporter gene GFP can be regulated in genetically modified fibroblasts in vitro and in vivo using a single retrovirus containing all elements for tetracyclineinducible gene expression. BDNF expression levels are dependent on the dose of doxycycline and can be rapidly increased or decreased in vitro by the addition or withdrawal of doxycycline, respectively. Compared with our previous studies (Blesch et al., 2000, 2001) using a similar retroviral vector harboring the tet-off system with the tTA (gene expression is turned off by doxycycline) instead of the rtTA, regulation increased from 36-fold to 100 - to 300 -fold in vitro. Although the baseline levels of expression without induction were three to six times higher and increased from $0.15 \mathrm{ng} / 10^{6}$ cells per $24 \mathrm{~h}$ to $0.5-1 \mathrm{ng} / 10^{6}$ cells per $24 \mathrm{~h}$, this "leakage" was not sufficient to result in biological responses such as de novo axonal growth in vivo. Comparison of the doxycycline sensitivity of the tet-on and tet-off systems also indicated that the dose of doxycycline needed to induce half-maximal expression in the current tet-on system was $\sim 1000$-fold higher $(\sim 30 \mathrm{ng} / \mathrm{ml})$ compared with the dose needed in our previous studies to reduce gene expression by $50 \%$ using the tet-off system $(20-30 \mathrm{pg} / \mathrm{ml}$ ) (Blesch et al., 2000). It is therefore possible that a higher dose of doxycycline administration in the drinking water would have resulted in 
higher levels of in vivo gene expression and doxycycline administration by systemic injection instead of oral intake might further improve regulation and limit some of the variability observed in the present set of experiments.

Regulated, transient expression of tropic factors delivered into a spinal cord lesion site could increase the probability of axonal bridging beyond the lesion site when combined with other treatments. Increased neurotrophin expression after peripheral nerve injury is known to play an important role during naturally occurring peripheral regeneration. Schwann cells transiently upregulate neurotrophic factor expression distal to a nerve lesion, and successful regeneration results in return of growth factor expression by Schwann cells to basal levels (Heumann et al., 1987; Funakoshi et al., 1993; Naveilhan et al., 1997; Trupp et al., 1997). A regulatable system such as that described in this report might be able to mimic such transient increases, supporting axonal bridging across a lesion site when combined with other suitable therapeutic interventions. A decline in neurotrophin production at the lesion site after axonal penetration has occurred, followed by an increase in neurotrophin production in the distal spinal cord, might enable axons to exit the graft and penetrate into distal host structures. In support of this possibility, our recent studies indicate that axons can bridge across a lesion site for short distances when growth factor gradients beyond the lesion site exist (Taylor et al., 2006).

\section{References}

Archelos JJ, Roggenbuck K, Schneider-Schaulies J, Linington C, Toyka KV, Hartung HP (1993) Production and characterization of monoclonal antibodies to the extracellular domain of P0. J Neurosci Res 35:46-53.

Bamber NI, Li H, Lu X, Oudega M, Aebischer P, Xu XM (2001) Neurotrophins BDNF and NT-3 promote axonal re-entry into the distal host spinal cord through Schwann cell-seeded mini-channels. Eur J Neurosci 13:257-268.

Blesch A, Tuszynski MH (2003) Cellular GDNF delivery promotes growth of motor and dorsal column sensory axons after partial and complete spinal cord transection, and induces remyelination. J Comp Neurol 467:403-417.

Blesch A, Uy HS, Grill RJ, Cheng JG, Patterson PH, Tuszynski MH (1999) LIF augments corticospinal axon growth and neurotrophin expression after adult CNS injury. J Neurosci 19:3556-3566.

Blesch A, Uy H, Diergardt N, Tuszynski MH (2000) Neurite outgrowth can be modulated in vitro using a tetracycline-repressible gene therapy vector expressing human nerve growth factor. J Neurosci Res 59:402-409.

Blesch A, Conner JM, Tuszynski MH (2001) Modulation of neuronal survival and axonal growth in vivo by tetracycline-regulated neurotrophin expression. Gene Ther 8:954-960.

Blesch A, Yang H, Weidner N, Hoang A, Otero D (2004) Axonal responses to cellularly delivered NT-4/5 after spinal cord injury. Mol Cell Neurosci 27:190-201.

Bradbury EJ, King VR, Simmons LJ, Priestley JV, McMahon SB (1998) NT-3, but not BDNF, prevents atrophy and death of axotomized spinal cord projection neurons. Eur J Neurosci 10:3058-3068.

Bradbury EJ, Khemani S, Von R, King, Priestley JV, McMahon SB (1999) NT-3 promotes growth of lesioned adult rat sensory axons ascending in the dorsal columns of the spinal cord. Eur J Neurosci 11:3873-3883.

Bunge MB, Kleitman N (1999) Neurotrophins and neuroprotection improve axonal regeneration into Schwann cell transplants placed in transected adult rat spinal cord. In: CNS regeneration: basic science and clinical applications (Tuszynski MH, Kordower JH, eds). San Diego: Academic.

Coumans JV, Lin TT, Dai HN, MacArthur L, McAtee M, Nash C, Bregman BS (2001) Axonal regeneration and functional recovery after complete spinal cord transection in rats by delayed treatment with transplants and neurotrophins. J Neurosci 21:9334-9344.

Funakoshi H, Frisen J, Barbany G, Timmusk T, Zachrisson O, Verge VM, Persson H (1993) Differential expression of mRNAs for neurotrophins and their receptors after axotomy of the sciatic nerve. J Cell Biol 123:455-465.
Gossen M, Bujard H (1992) Tight control of gene expression in mammalian cells by tetracycline-responsive promoters. Proc Natl Acad Sci USA 89:5547-5551.

Gossen M, Bonin AL, Bujard H (1993) Control of gene activity in higher eukaryotic cells by prokaryotic regulatory elements. Trends Biochem Sci 18:471-475.

Gossen M, Freundlieb S, Bender G, Muller G, Hillen W, Bujard H (1995) Transcriptional activation by tetracyclines in mammalian cells. Science 268:1766-1769.

Grill R, Murai K, Blesch A, Gage FH, Tuszynski MH (1997) Cellular delivery of neurotrophin-3 promotes corticospinal axonal growth and partial functional recovery after spinal cord injury. J Neurosci 17:5560-5572.

Hebb DO (1949) The organization of behavior. New York: Wiley.

Heumann R, Lindholm D, Bandtlow C, Meyer M, Radeke MJ, Misko TP, Shooter E, Thoenen H (1987) Differential regulation of mRNA encoding nerve growth factor and its receptor in rat sciatic nerve during development, degeneration, and regeneration: role of macrophages. Proc Natl Acad Sci USA 84:8735-8739.

Himes BT, Liu Y, Solowska JM, Snyder EY, Fischer I, Tessler A (2001) Transplants of cells genetically modified to express neurotrophin-3 rescue axotomized Clarke's nucleus neurons after spinal cord hemisection in adult rats. J Neurosci Res 65:549-564.

Houle JD, Jin Y (2001) Chronically injured supraspinal neurons exhibit only modest axonal dieback in response to a cervical hemisection lesion. Exp Neurol 169:208-217.

Houle JD, Ye JH (1999) Survival of chronically-injured neurons can be prolonged by treatment with neurotrophic factors. Neuroscience 94:929-936.

Jakeman LB, Wei P, Guan Z, Stokes BT (1998) Brain-derived neurotrophic factor stimulates hindlimb stepping and sprouting of cholinergic fibers after spinal cord injury. Exp Neurol 154:170-184.

Jin Y, Tessler A, Fischer I, Houle JD (2000) Fibroblasts genetically modified to produce BDNF support regrowth of chronically injured serotonergic axons. Neurorehabil Neural Repair 14:311-317.

Jin Y, Fischer I, Tessler A, Houle JD (2002) Transplants of fibroblasts genetically modified to express BDNF promote axonal regeneration from supraspinal neurons following chronic spinal cord injury. Exp Neurol 177:265-275.

Kobayashi NR, Fan DP, Giehl KM, Bedard AM, Wiegand SJ, Tetzlaff W (1997) BDNF and NT-4/5 prevent atrophy of rat rubrospinal neurons after cervical axotomy, stimulate GAP- 43 and T $\alpha 1$-tubulin mRNA expression, and promote axonal regeneration. J Neurosci 17:9583-9595.

Kwon BK, Liu J, Messerer C, Kobayashi NR, McGraw J, Oschipok L, Tetzlaff W (2002) Survival and regeneration of rubrospinal neurons 1 year after spinal cord injury. Proc Natl Acad Sci USA 99:3246-3251.

Liu Y, Kim D, Himes BT, Chow SY, Schallert T, Murray M, Tessler A, Fischer I (1999) Transplants of fibroblasts genetically modified to express brain-derived neurotrophic factor promote regeneration of adult rat rubrospinal axons and recovery of forelimb function. J Neurosci 19:4370-4387.

Lu P, Blesch A, Tuszynski MH (2001) Neurotrophism without neurotropism: BDNF promotes survival but not growth of lesioned corticospinal neurons. J Comp Neurol 436:456-470.

Lu P, Jones LL, Tuszynski MH (2005) BDNF-expressing marrow stromal cells support extensive axonal growth at sites of spinal cord injury. Exp Neurol 191:344-360.

Ma L, Harada T, Harada C, Romero M, Hebert JM, McConnell SK, Parada LF (2002) Neurotrophin-3 is required for appropriate establishment of thalamocortical connections. Neuron 36:623-634.

Maguire-Zeiss KA, Federoff HJ (2004) Safety of viral vectors for neurological gene therapies. Curr Opin Mol Ther 6:473-481.

Markus A, Patel TD, Snider WD (2002) Neurotrophic factors and axonal growth. Curr Opin Neurobiol 12:523-531.

Marotte LR, Vidovic M, Wheeler E, Jhaveri S (2004) Brain-derived neurotrophic factor is expressed in a gradient in the superior colliculus during development of the retinocollicular projection. Eur J Neurosci 20:843-847.

Menei P, Montero-Menei C, Whittemore SR, Bunge RP, Bunge MB (1998) Schwann cells genetically modified to secrete human BDNF promote enhanced axonal regrowth across transected adult rat spinal cord. Eur J Neurosci 10:607-621.

Naveilhan P, ElShamy WM, Ernfors P (1997) Differential regulation of mR- 
NAs for GDNF and its receptors Ret and GDNFR alpha after sciatic nerve lesion in the mouse. Eur J Neurosci 9:1450-1460.

Pear WS, Scott ML, Nolan GP (1997) Generation of high-titer, helper-free retroviruses by transient transfection. In: Methods in molecular medicine: gene therapy protocols (Robbins PD, ed), pp 41-57. Totowa, NJ: Humana.

Ramer MS, Priestley JV, McMahon SB (2000) Functional regeneration of sensory axons into the adult spinal cord. Nature 403:312-316.

Taylor L, Jones L, Tuszynski MH, Blesch A (2006) Neurotrophin-3 gradients established by lentiviral gene delivery promote short-distance axonal bridging beyond cellular grafts in the injured spinal cord. J Neurosci 26:9713-9721.

Tessier-Lavigne M, Goodman CS (1996) The molecular biology of axon guidance. Science 274:1123-1133.

Tobias CA, Shumsky JS, Shibata M, Tuszynski MH, Fischer I, Tessler A, Murray M (2003) Delayed grafting of BDNF and NT-3 producing fibroblasts into the injured spinal cord stimulates sprouting, partially rescues axotomized red nucleus neurons from loss and atrophy, and provides limited regeneration. Exp Neurol 184:97-113.

Trupp M, Belluardo N, Funakoshi H, Ibanez CF (1997) Complementary and overlapping expression of glial cell line-derived neurotrophic factor (GDNF), c-ret proto-oncogene, and GDNF receptor- $\alpha$ indicates multiple mechanisms of trophic actions in the adult rat CNS. J Neurosci 17:3554-3567.

Tucker KL, Meyer M, Barde YA (2001) Neurotrophins are required for nerve growth during development. Nat Neurosci 4:29-37.

Tuszynski MH, Peterson DA, Ray J, Baird A, Nakahara Y, Gage FH (1994) Fibroblasts genetically modified to produce nerve growth factor induce robust neuritic ingrowth after grafting to the spinal cord. Exp Neurol 126:1-14.

Tuszynski MH, Gabriel K, Gage FH, Suhr S, Meyer S, Rosetti A (1996) Nerve growth factor delivery by gene transfer induces differential outgrowth of sensory, motor, and noradrenergic neurites after adult spinal cord injury. Exp Neurol 137:157-173.

Tuszynski M, Grill R, Blesch A (1999) Spinal cord regeneration. In: CNS regeneration: basic science and clinical applications (Tuszynski M, Kordower J, eds), pp 605-630. San Diego: Academic.

Urlinger S, Baron U, Thellmann M, Hasan MT, Bujard H, Hillen W (2000) Exploring the sequence space for tetracycline-dependent transcriptional activators: novel mutations yield expanded range and sensitivity. Proc Natl Acad Sci USA 97:7963-7968.

Weidner N, Blesch A, Grill RJ, Tuszynski MH (1999) Nerve growth factorhypersecreting Schwann cell grafts augment and guide spinal cord axonal growth and remyelinate central nervous system axons in a phenotypically appropriate manner that correlates with expression of L1. J Comp Neurol 413:495-506.

Xu XM, Guenard V, Kleitman N, Aebischer P, Bunge MB (1995) A combination of BDNF and NT-3 promotes supraspinal axonal regeneration into Schwann cell grafts in adult rat thoracic spinal cord. Exp Neurol 134:261-272.

Ye JH, Houle JD (1997) Treatment of the chronically injured spinal cord with neurotrophic factors can promote axonal regeneration from supraspinal neurons. Exp Neurol 143:70-81.

Zoltick PW, Wilson JM (2001) Regulated gene expression in gene therapy. Ann NY Acad Sci 953:53-63. 